\title{
Anisotropic Behavior of Radiopaque NiTiPt Hypotube for Biomedical Applications
}

\author{
By Zhicheng Lin, Hao-Ming Hsiao, ${ }^{*}$ David Mackiewicz, Boris Anukhin and Kelly Pike
}

Nitinol (NiTi) has become one of the most important implant materials in biomedical applications such as endovascular stents, embolic protection filters, and endodontic files. However, NiTi stents sometimes do not provide satisfactory visibility under fluoroscopy during angioplasty. A new ternary alloy, radiopaque nitinol (NiTiPt), exhibits much better radiopacity than NiTi and has great potential for future biomedical applications. This paper presents the first characterization of anisotropic stress-strain behavior in micron-sized specimens cut directly from hypotubes of this new alloy NiTiPt. Experimental results show that NiTiPt hypotube has very different anisotropic characteristics and high stress-strain nonlinearity when compared to its NiTi counterpart. Its ultimate tensile strength and strain are approximately 15-40\% higher than NiTi with statistically significant difference for all three cutting angles measured. Its stress-strain behavior has several unique features including lower initial austenite elastic modulus, smaller hysteresis loop, and sharper tails during the loading-unloading cycle. The $45^{\circ}$ behavior is particularly different from other angles, with stronger hardening effect and an approximately $25-30 \%$ reduction in ultimate strain when compared to $0^{\circ}$.

NiTi stent is a small, coiled wire-mesh tube that can be deployed into a blood vessel and self-expanded during angioplasty to open a narrowed blood vessel (Fig. 1). The expanded stent exerts radial force against the walls of the artery, thereby preventing reclosure of the artery. However, NiTi stents may not provide satisfactory visibility under fluoroscopy during angioplasty due to its narrow and thin wire mesh, usually in the order of $100-150 \mu \mathrm{m}$. NiTiPt, a new ternary alloy, consists of three elements: nickel, titanium, and platinum, with more radiopaque platinum replacing certain percentage of nickel. ${ }^{[1]}$ Figure 2 shows the radiopacity comparison between NiTiPt and NiTi stents. The top five stents in Figure 2 are NiTiPt stents with various weights from light to heavy, while the bottom two stents are NiTi stents. The markers on the very bottom NiTi stent were made from the

[*] Dr. H. Hsiao

Department of Mechanical Engineering,

National Taiwan University, Taipei, Taiwan

E-mail: hmhsiao@ntu.edu.tw

Dr. Z. Lin, D. Mackiewicz, B. Anukhin, K. Pike

Abbott Laboratories, Abbott Vascular, Santa Clara,

CA 95054, USA
NiTiPt material. It appears that NiTiPt stent has higher radiopacity than NiTi stent.

$\mathrm{NiTi}$ is shown to have anisotropic characteristics. Processing techniques such as heat-set treatment, sheet rolling, or tube drawing used in the production of biomedical NiTi devices can lead to texturing (crystallographic alignment). There are a few references regarding texture in NiTi sheets and plates but very limited information in NiTi hypotubes, the starting material for manufacturing endovascular stents and other biomedical devices. ${ }^{[2-5]}$ Since the new radiopaque NiTiPt stent requires heat-set treatments to optimize its properties, the very same question is asked: Is the NiTiPt stent anisotropic?

Anisotropic characteristics could have critical effects in modeling the biomedical NiTi device performance using finite element analysis (FEA). FEA is an increasingly important tool to obtain rapid evaluation of the stent acute performance and predict the stent long-term fatigue resistance. ${ }^{[6-8]}$ However, most of the FEA models in the medical device industry to date have made the assumption that NiTi is isotropic. Such an assumption could impact the accuracy of the FEA predictions for biomedical NiTi devices. In order to accurately predict the NiTi device performance, anisotropic stress-strain behavior in the product form of rod, sheet, or tube has to be better understood and fully characterized.

In this paper, the tested specimens were cut directly from hypotubes in a variety of angles to the hypotube drawing direction and then subjected to the same heat-set treatment and electro-polishing procedures used for manufacturing stents to ensure that the specimens captured the anisotropic characteristics of an actual stent cut from a hypotube. The anisotropic behavior of both NiTiPt and NiTi hypotubes were investigated. The effects of the orientation on the material

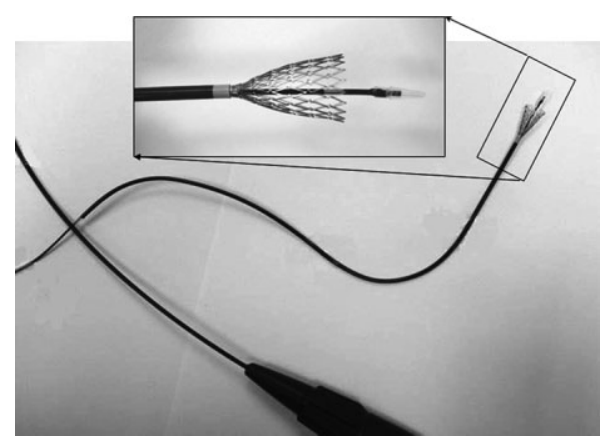

Fig. 1. A self-expanding NiTi stent released from catheter for deployment. 


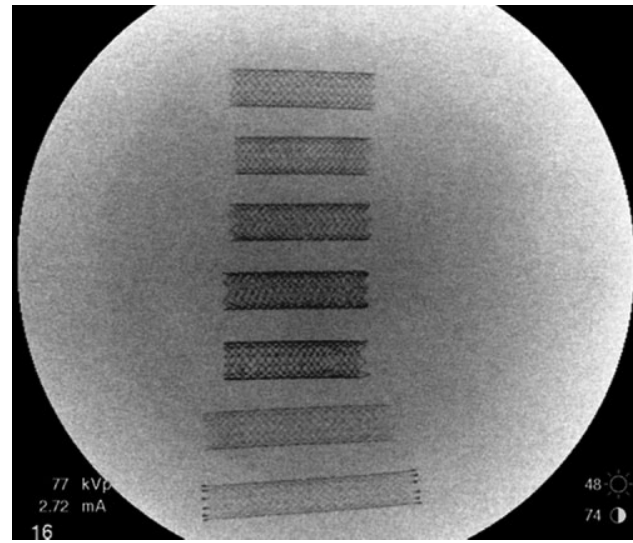

Fig. 2. Radiopacity comparison between NiTiPt stents (top five) and NiTi stents (bottom two).

behavior such as ultimate tensile strength and strain, hysteresis loop, and shape of the stress-strain curves were analyzed experimentally.

\section{Specimen Preparation and Experimental Setup}

Intravascular stents were manufactured by laser cutting of the hypotubes with a laser beam size as small as a few micrometer (Fig. 3), followed by multiple heat-set treatments to "train" the stents to the desired configuration. In order to study the stress-strain behavior in a variety of angles to the hypotube drawing direction, tensile dogbone specimens were prepared with angles varied from along the drawing direction (longitudinal) to almost perpendicular to the drawing direction (circumferential). Laser cutting, heat-set treatment, mechanical, chemical, and electro-polishing procedures, identical to the stent manufacturing process, were implemented on these tested specimens. The following steps describe the procedures of preparing tested specimens with various cutting angles:

(1) Laser cut the spiral specimens from hypotubes (Fig. 4): The angle $\alpha$ of the spiral specimens was 0,45 , and $82^{\circ}$. Both NiTiPt and NiTi hypotubes were obtained from the same supplier for consistency. All tubes had the same outer diameter of $3.3 \mathrm{~mm}$ and the same thickness of $280 \mu \mathrm{m}$ for direct comparison. NiTiPt dogbone samples had the $A_{\mathrm{f}}$ of

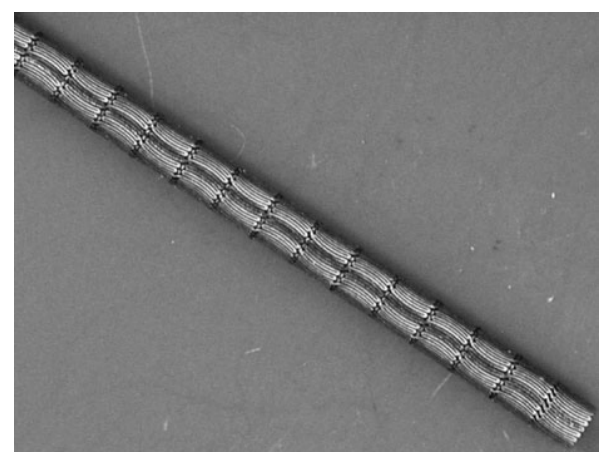

Fig. 3. An intravascular stent laser cut from a hypotube.

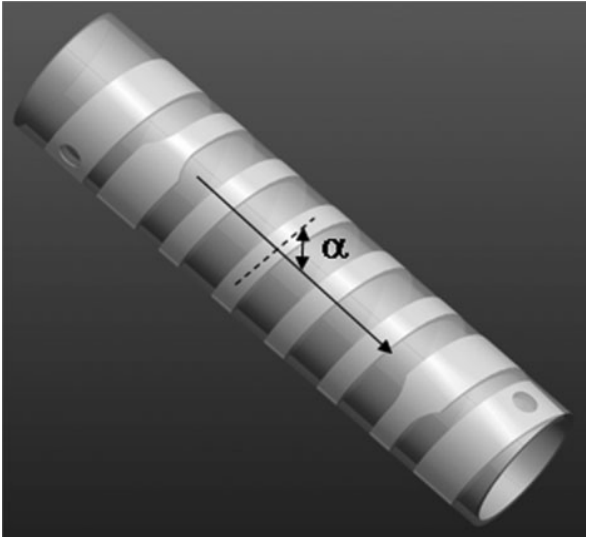

Fig. 4. A Spiral specimen laser cut from a hypotube (black arrow: drawing direction of the tube; dash line: spine line of the specimen; angle $\alpha$ : $\left.0-82^{\circ}\right)$.

$0{ }^{\circ} \mathrm{C}$, while NiTi dogbone samples had the $A_{\mathrm{f}}$ of $15^{\circ} \mathrm{C}$. The $A_{\mathrm{f}}$ temperatures were measured by the bend and free recovery (BFR) method in accordance with ASTM F 2082-03 after the samples were heat set to their final shapes. Both NiTi and NiTiPt samples were tested in body temperature at $37^{\circ} \mathrm{C}$ which is above the austenite finish temperature.

(2) Flattened the spiral specimens into the tested specimens: This process included unrolls and flattening of the spiral specimens inside a fixture, followed by multiple heat-set treatments at $530{ }^{\circ} \mathrm{C}$ for $2 \mathrm{~min}$. This process removed the residual stresses in the spiral specimens. The temperature $\left(530^{\circ} \mathrm{C}\right)$ was used for heat-setting the stents and by subjecting the tested specimens under the same processing conditions, they were able to develop similar anisotropic characteristics representing an actual stent. Specimens cut along the longitudinal direction were already straight after laser cut; however, they were also subjected to the same heat-set treatments as other specimens to ensure consistent thermal history across the groups.

(3) Electro-polished the tested specimens to ensure the surface finished quality consistent with the final stent products: A finished dogbone specimen had the gage dimensions of $400 \mu \mathrm{m}$ wide, $200 \mu \mathrm{m}$ thick, and $45 \mathrm{~mm}$ long. The outer diameter of the tubing was $3.3 \mathrm{~mm}$ and therefore, the largest possible angle $\alpha$ of a spiral specimen was $82^{\circ}$. Tensile testing was conducted on an INSTRON electromechanical test system configured with a $100 \mathrm{~N}$ load cell. The INSTRON standard video extensometer was used with a $100 \mathrm{~mm}$ field of view lens and $500 \mathrm{~mm}$ lighting array. The marks used for tracking deformation with the video were $2 \mathrm{~mm}$ wide black pieces of tape that were wrapped around the specimen at a distance approximately $25 \mathrm{~mm}$ apart. Each specimen was pulled to $6 \%$ strain, unloaded to $3.5 \mathrm{MPa}$, and then pulled again to failure.

Results

Stress-strain behavior was characterized on specimens cut from a variety of angles to the hypotube drawing direction for 

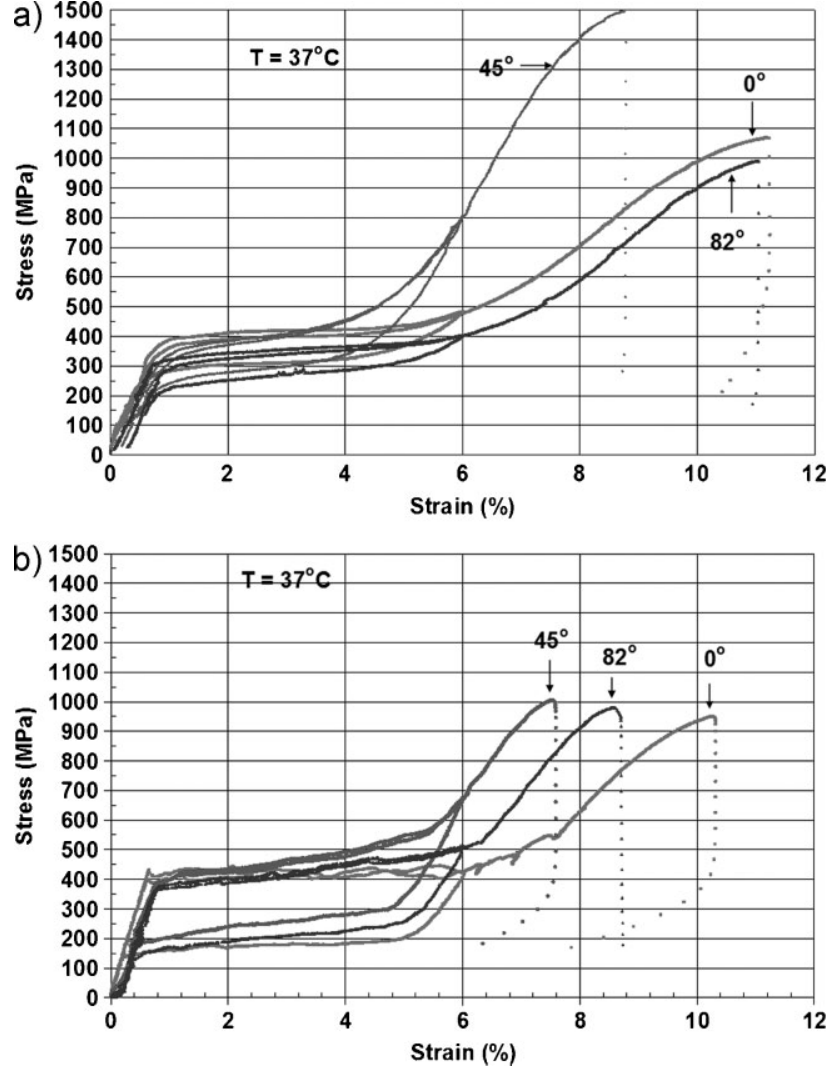

Fig. 5. Stress-strain curves of dogbone specimens with various angles from 0 to $82^{\circ}$ at $37^{\circ} \mathrm{C}$ : (a) NiTiPt and (b) NiTi.

both NiTiPt and NiTi at the body temperature of $37^{\circ} \mathrm{C}$ (Fig. 5). Since the specimens were subjected to the same laser cutting, heat-set treatment, and electro-polishing procedures used for manufacturing stents, it is believed these tested specimens were able to capture the anisotropic characteristics of an actual stent. Table 1 summarizes the comparison between NiTiPt and NiTi for ultimate tensile strength and strain at various cutting angles. A total of five to six specimens were tested for each angle. The standard deviation was relatively low for 0 and $82^{\circ}$ but slightly higher for $45^{\circ}$. The $P$ values from the $t$-test were small, indicating there is statistically significant difference between the NiTiPt and NiTi hypotube properties.

Both NiTiPt and NiTi hypotubes exhibit strong material anisotropy. They appear to follow the trend of being more ductile at $0^{\circ}$, then becoming most brittle at $45^{\circ}$, and finally returning to ductile at $82^{\circ}$. This trend is different from the stress-strain behavior observed in NiTi sheets with $20 \%$ cold work, ${ }^{[9]}$ where the 0 and $90^{\circ}$ (from the sheet rolling direction) are more brittle and the $22.5^{\circ}$ is the most ductile. This difference implies that each product form (rod, sheet, or tube) has its unique characteristic textures and therefore, sheet data should not be used as a substitute for hypotubes or stents. The $45^{\circ}$ behavior is particularly different from other angles, with stronger hardening effect and an approximately 25-30\% reduction in ultimate strain when compared to $0^{\circ}$. The significant drop of the ultimate strain at $45^{\circ}$ seems to agree well with observation that stent fractures in fatigue testing often occur along $45^{\circ}$ from the hypotube drawing direction.

Figure 6 reveals the microstructures and grain sizes of the NiTiPt and NiTi stents. Both have polycrystalline and micro-/ nano-grained microstructure with the NiTiPt stent showing finer grain size than the NiTi stent. Their grains have the oval shape elongated along the drawing direction, suggesting both materials exhibit higher degree of anisotropy and have preferential orientation. This confirms the observation from the stress-strain characterization.

\section{Discussions}

The NiTiPt hypotube shows very different anisotropic characteristics and high stress-strain nonlinearity when compared to its NiTi counterpart (Fig. 7). The shape of the stress-strain curves and the hysteresis loop are different. NiTiPt initial elastic modulus at the austenite stage is lower for all angles. However, its stress-induced martensite elastic modulus stiffens up considerably and its ultimate tensile strength and strain are statistically significantly higher than NiTi by approximately $15-40 \%$. The findings support the hypothesis that NiTiPt alloy is of great value for future biomedical applications with special regard to novel techniques such as percutaneous stent-valve procedures which require optimized stress-strain behavior. NiTiPt stress-strain behavior has other unique features which are different from $\mathrm{NiTi}$; it has lower martensitic transformation upper-plateau stress and higher martensitic transformation lower-plateau stress, thus forming a much smaller hysteresis loop than NiTi. The smaller hysteresis loop represents lower energy absorption during the loading-unloading cycle and less damping effect of the material. NiTiPt also has sharper tails during

Table 1. Comparison between NiTiPt and NiTi for Ultimate Tensile Strength and Strain at $37^{\circ} \mathrm{C}$.

\begin{tabular}{|c|c|c|c|c|c|c|}
\hline & \multirow[t]{2}{*}{ Angle } & \multicolumn{2}{|c|}{ NiTiPt } & \multicolumn{2}{|c|}{$\mathrm{NiTi}$} & \multirow[t]{2}{*}{$P(t$-test $)$} \\
\hline & & Average & S.D & Average & S.D. & \\
\hline \multirow[t]{3}{*}{ Ultimate Tensile Strength (MPa) } & $0^{\circ}$ & $1070.2 \mathrm{MPa}$ & $18.9 \mathrm{MPa}$ & $839.2 \mathrm{MPa}$ & $58.0 \mathrm{MPa}$ & 0.0001 \\
\hline & $45^{\circ}$ & $1200.0 \mathrm{MPa}$ & $167.4 \mathrm{MPa}$ & $932.6 \mathrm{MPa}$ & $133.0 \mathrm{MPa}$ & 0.0185 \\
\hline & $82^{\circ}$ & $998.5 \mathrm{MPa}$ & $46.0 \mathrm{MPa}$ & $861.6 \mathrm{MPa}$ & $90.9 \mathrm{MPa}$ & 0.0110 \\
\hline \multirow{3}{*}{ Ultimate Tensile Strain (\%) } & $0^{\circ}$ & $11.6 \%$ & $0.3 \%$ & $10.1 \%$ & $0.2 \%$ & 0.0000 \\
\hline & $45^{\circ}$ & $8.7 \%$ & $0.6 \%$ & $7.5 \%$ & $0.4 \%$ & 0.0043 \\
\hline & $82^{\circ}$ & $11.3 \%$ & $0.4 \%$ & $8.0 \%$ & $0.6 \%$ & 0.0000 \\
\hline
\end{tabular}



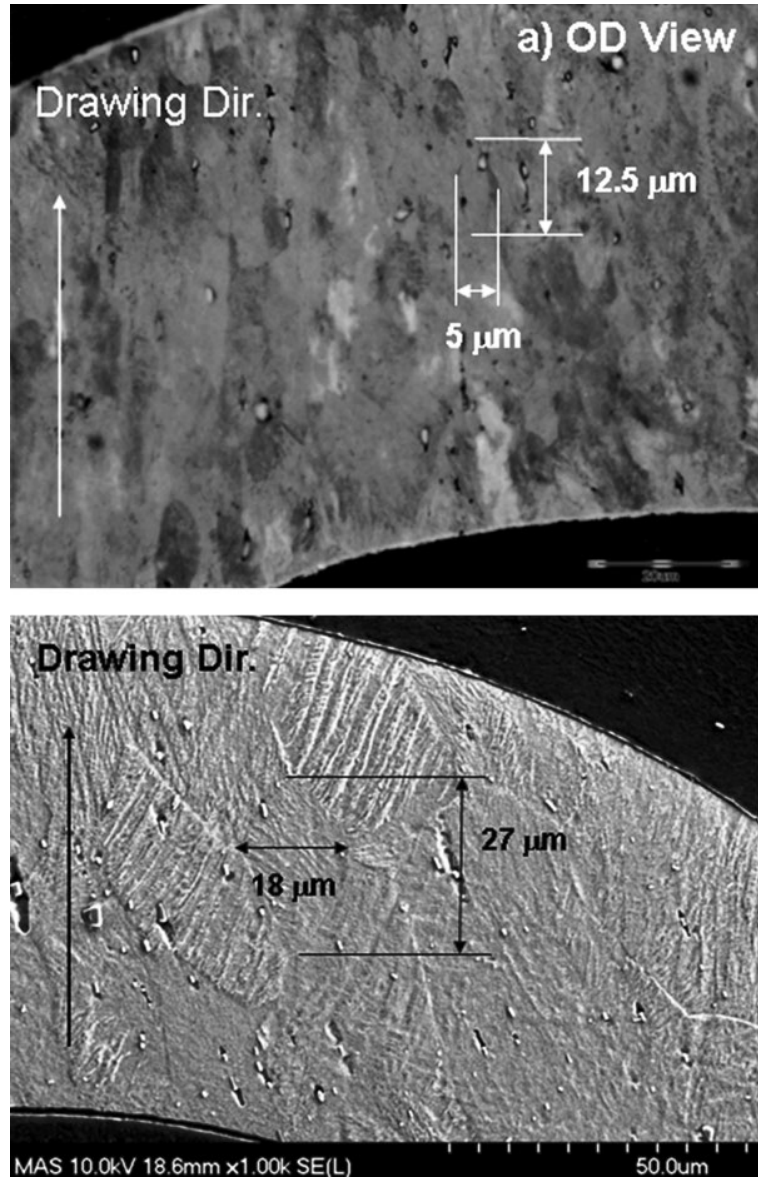

Fig. 6. Stent microstructure from the tubing side view: (a) NiTiPt and (b) NiTi.

loading-unloading so its recoverable pseudoelastic transformation strain is not that well defined as NiTi.

The $A_{\mathrm{f}}$ temperature of the NiTiPt dogbone samples is around $0{ }^{\circ} \mathrm{C}$, while the $A_{\mathrm{f}}$ temperature of the NiTi dogbone samples is around $15^{\circ} \mathrm{C}$. Both NiTi and NiTiPt samples were tested in body temperature at $37^{\circ} \mathrm{C}$ in their austenitic phase. Since the samples were subjected to heat treatments before testing, the $A_{\mathrm{f}}$ temperature may change significantly within minutes as suggested by other authors, ${ }^{[10,11]}$ a fact that shows the complexity of this topic concerning the influence of $A_{\mathrm{f}}$ on material behavior. The difference in $A_{\mathrm{f}}$ could affect the plateau stresses and permanent set in the stress-stain loops; however, it is unlikely this would change the anisotropic characteristics of both alloys found in this study.

The observed anisotropic behavior in NiTiPt and $\mathrm{NiTi}$ could impact the FEA predictions for biomedical NiTiPt or NiTi devices. In order to accurately predict the device performance, anisotropic stress-strain behavior in the product forms (rod, sheet, or tube) has to be fully characterized and implemented in FEA models. In addition, in the current device industry, most of the coronary and endovascular stent FEA models are assessed using the maximum stress-strain criteria with the assumption that the material properties are isotropic. However, since both NiTiPt and NiTi hypotubes exhibit strong anisotropy, anisotropic yield criteria such as the
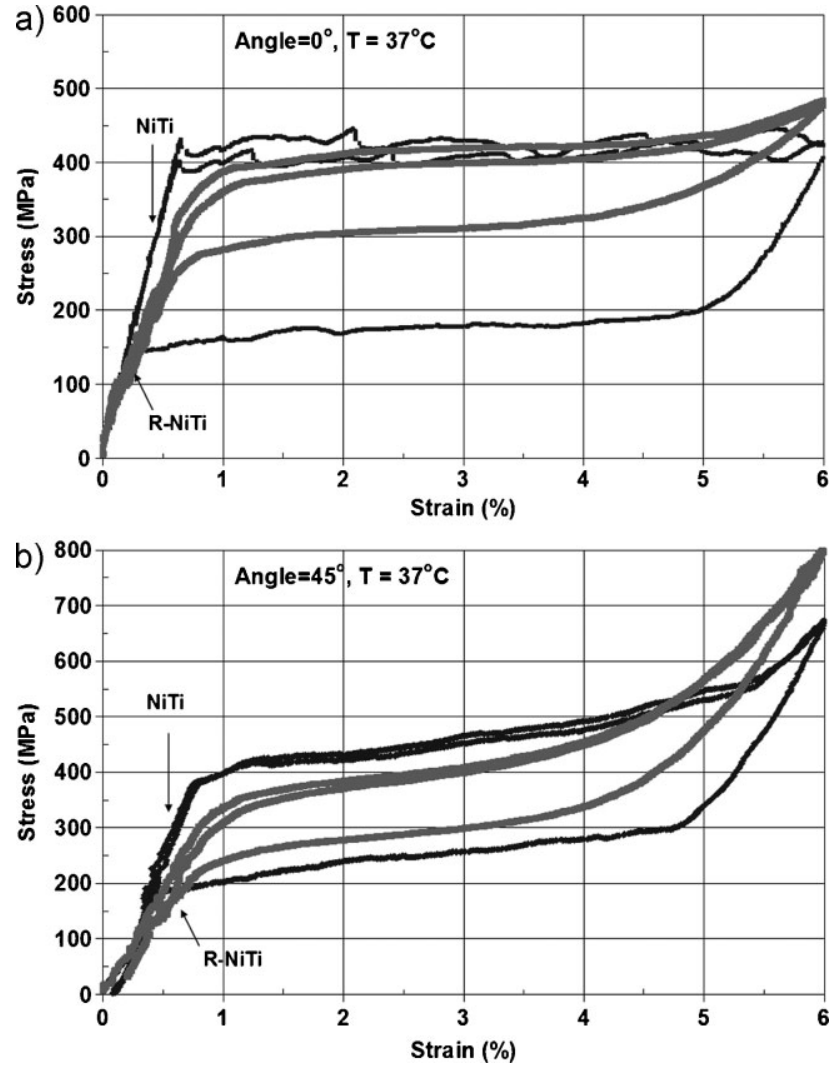

Fig. 7. Comparison of stress-strain curves up to $6 \%$ between NiTiPt and NiTi at $37^{\circ} \mathrm{C}$ : (a) $0^{\circ}$ and (b) $45^{\circ}$

following multi-axial interactive tensor polynomial in the strain space may be needed in the future to account for the material anisotropy and predict the NiTiPt or NiTi device failure

$F_{i} \varepsilon_{i}+F_{i j} \varepsilon_{i} \varepsilon_{j}=1, \quad i, j=1-6$

where $F_{i}$ and $F_{i j}$ are strain tensors of the second and fourth rank, respectively.

\section{Conclusions}

This paper presents the first characterization of anisotropic stress-strain behavior in micron-sized specimens cut directly from hypotubes, the starting material for the manufacture of endovascular stents and other biomedical devices, of a new alloy NiTiPt. The specimens were subjected to the same heat-set treatment and electro-polishing procedures used for manufacturing stents to ensure that the tested specimens captured the anisotropic characteristics of an actual stent cut from a hypotube. Experimental results show that NiTiPt hypotube has very different anisotropic characteristics and high stress-strain nonlinearity when compared to its NiTi counterpart. Its ultimate tensile strength and strain are approximately $15-40 \%$ higher than NiTi with statistically significant difference for all three cutting angles measured. Its stress-strain behavior has several unique features including lower initial austenite elastic modulus, smaller hysteresis 
loop, and sharper tails during loading-unloading cycle. It is recommended that, in order to accurately predict the NiTiPt or NiTi device performance, anisotropic stress-strain behavior in the product forms (rod, sheet, or tube) has to be fully characterized and implemented in FEA models. Anisotropic yield criteria such as the multi-axial interactive tensor polynomial in the strain space may be needed in the future for the NiTiPt or NiTi device failure prediction.

Received: November 25, 2008

Final Version: June 8, 2009

Published online: August 24, 2009

[1] J. F. Boylan, in Proceedings International Conference on Shape Memory and Superelastic Technologies, ASM International, Materials Park OH 2003, 1.

[2] J. H. Mulder, P. E. Thoma, J. Beyer, Z. Metallkd. 1993, 84, 501.

[3] H. Inoue, N. Miwa, N. Inakazu, Acta Mater. 1996, 44, 4825 .
[4] S. Gao, S. Yi, Mater. Sci. Eng. 2003, A362, 107.

[5] S. W. Robertson, V. Imbeni, E. Notkina, H. R. Wenk, R. O. Ritchie, J. Biomed. Mater. Res.: Part A 2005, 72A, 190.

[6] H. M. Hsiao, S. Prabhu, A. Nikanorov, M. Razavi, ASME J. Med. Devices 2007, 1, 113.

[7] S. W. Robertson, R. O. Ritchie, Biomaterials 2007, 28, 700.

[8] H. M. Hsiao, A. Nikanorov, S. Prabhu, M. Razavi, J. Biomed. Mater. Res.: Part B 2009, DOI: 10.1002/ jbm.b.31424 (in press)

[9] Z. C. Lin, J. F. Boylan, in Proceedings International Conference on Shape Memory and Superelastic Technologies, ASM International, Materials Park OH 2001, 313.

[10] M. J. Drexel, G. S. Selvaduray, A. R. Pelton, in Proceedings International Conference on Shape Memory and Superelastic Technologies, ASM International, Materials Park $\mathrm{OH}$ 2008, 447.

[11] A. Undisz, M. Fink, M. Rettenmayr, Scr. Mater. 2008, 59, 979.

\section{Interference Lithography - a new powerful technique}

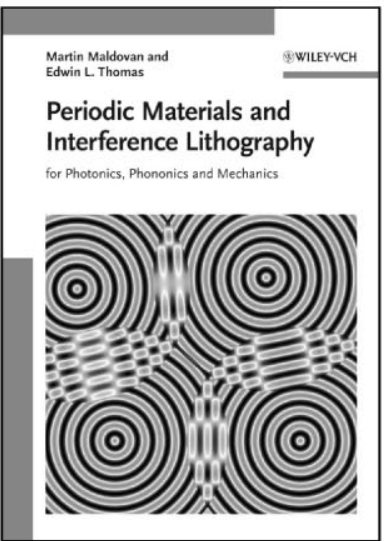

2008. XVIII, 313 pages, 118 figures 3 in color, 19 tables. Hardcover.

ISBN: 978-3-527-31999-2 $€ 119 .-/ £ 105 .-/ U S \$ 165$.
MARTIN MALDOVAN, EDWIN L. THOMAS

both of Massachusetts Institute of Technology, Cambridge, USA

\section{Periodic Materials and Interference Lithography} for Photonics, Phononics and Mechanics

Written by Martin Maldovan and Edwin Thomas, head of MIT's Department of Materials Science and Engineering, this concise and stringent introduction takes readers from the fundamental theory, via experimentation, to in-depth knowledge. A must-have for both beginners and veterans in the field.
Register now for the free WILEY-VCH Newsletter! www.wiley-vch.de/home/pas
WILEY-VCH •P.O. Box $101161 \cdot$ D-69451 Weinheim, Germany

Fax: +49 (0) $6201-606184$

e-mail: service@wiley-vch.de•http://nww.wiley-vch.de 